\title{
Micro-patterned plasma polymer films for bio-sensing
}

\author{
Marianne Vandenbossche* ${ }^{* 1}$,Laetitia Bernard ${ }^{2}$, Patrick Rupper $^{1}$, Katharina Maniura-Weber ${ }^{3}$,Manfred \\ Heuberger $^{1}$, Greta Faccio ${ }^{3}$,Dirk Hegemann ${ }^{1}$ \\ ${ }^{1}$ Laboratory for Advanced Fibers, Empa, Swiss Federal Laboratories for Materials Science and Technology, \\ Lerchenfeldstrasse 5, 9014St. Gallen, Switzerland \\ ${ }^{2}$ Laboratory for Nanoscale Materials Science, Empa, Swiss Federal Laboratories for Materials Science and \\ Technology, Überlandstrasse 129, 8600 Dübendorf, Switzerland \\ ${ }^{3}$ Laboratory for Biointerfaces, Empa, Swiss Federal Laboratories for Materials Science and Technology, \\ Lerchenfeldstrasse 5, 9014 St. Gallen, Switzerland.
}

*Corresponding Author: E-mail: marianne.vandenbossche@empa.ch

\begin{abstract}
Bi-functional micro-patterned plasma polymer films were prepared by first depositing afull-area amino-containing plasma polymer film (PPF) onto a substrate, followed bythe deposition ofa patterned oxygen-containing PPF viaa polymeric mesh used as contact mask. As a result, an ultrathin film with alternating areas of amine and oxygen-containing groupsis obtained. Such micro-patterns bear potential as templates for multi-sensitive bio-sensor substrates.Thechemical surface composition is assessed using XPS and ToF-SIMS. On the masked amino-rich areaa lateral chemical micro gradient was observed due to mesh mask shadowing. Stability of the coating was investigated over time in ambient air and in water, showing that the surface properties were maintained after 7 days in air and 1 days in water.To demonstrate functionality, the micro-patterned surface was exposed toa green fluorescent protein (GFP) andlateral resolution and selectivity during adsorption was studied.
\end{abstract}

\section{KEYWORDS}

Micro-patterned polymer films; plasma deposition; surface functionalization; film characterization; protein adsorption. 


\section{INTRODUCTION}

The use of plasma-based coatings for biomedical purposes is a growing field. As these coatings are prepared by asolventless and eco-friendly plasma deposition method, it is possible to provide surfaces able to favor cell cultures [1-3] as well as surfaces that can be used for bio-sensing. [4,5]Optical bio-sensing devices, such as Surface PlasmonResonance (SPR) sensor [4] or Transmission Interferometric Adsorption Sensor (TInAS) [5], can be used to detect adsorption of biological ligands from complex fluids. These label-free detection devices are based on affinity interactions between biomarkers and immobilized biomolecules, such as antibodies, proteins, lipids or DNA [6,7]. Surface preparation of the biosensor is thus crucial in order to ensure the reproducibility of the quantifiedresults when analyzing biological fluids.

Biomolecules can be covalently immobilizedto the surface of the sensor utilizing polymeric films or hydrogelsmadebymolecular assembly as host matrix. More recently, molecular assemblyhas attracted increased attention as it allows patterning with two or more functional properties:for example, enhancement of the specific recognition of a biomolecule and avoidance of the nonspecific adsorption of other compounds.For example, such surfaces were obtained by molecular assembly at micro-[8] and nanoscale [9], where biomolecules responsible for the affinity of the sensor were immobilized on the terminal functional groups of poly(L-lysine)-graft-poly(ethylene glycol) copolymers, which provide a hydrated brushtype coating that can avoid non-specific adsorption [10]. These methods have in common that organic solvents are required during surface preparation. A more eco-friendly and industry transferablemethod to preparemicro-patterned polymer films via plasma processing is presented here.

Plasma processing of sensorsurfacesis accompanied by a number of key advantages such as i n-situ pre-cleaning and subsequent polymerization or functionalization steps. Furthermore, plasma deposition readily forms flat and smooth coatings at full surface coverage. Thanks to previous studies on such plasma depositions [11-14], the thickness of the deposited films, number of accessible chemical groups and cross-linking degree (e.g. stability) can be controlled.

For a micro-patterned plasma polymer film ( $\mu$ p-PPF)produced in-situ,be it in alowpressure plasma reactor [15-17] or in an atmospheric plasma [18,19], a number of sequential approacheshave already been investigated [20].Typically, they consist ofeither etching a polymeric surfaceor depositinga polymer film through a mask [15-18,21-23]. Another 
established methodto obtain micro-patterned chemical functionalization is colloidal lithography or colloidal nanolithography, where the etching or the deposition occurs between adsorbed particlesthat forma colloidal mask [23-28].Some plasma reactors are able to produce some degree of micro-patterning thanks to a sophisticated geometry of the used electrode; e.g.a microplasma device exploiting plasma polymerization through asymmetric glow discharge [19,29-31]. Other methods consist of depositing a plasma polymer film onto a substrate, then creating a micro-patternthanks to lithography [32] or nano-lithography [33].

The aim of this paper is to propose a new type of micro-patterned coating with spatially defined distinct functional groupsthat is generated in-situ during a plasma process. To achieve this goal, two different plasma polymer films PPFs, i.e. amino-containing (a$\mathrm{C}: \mathrm{H}: \mathrm{N})$ and oxygen-containing (a-C:H:O) PPFs, were partially overlaid at the surface of the substrate. Such PPFs were selected due to their general interest as biointerfaces as well as previous own work [34].The micro-patterning was obtained in an innovative mannerby the use of a monofilament mesh mask during the second a-C:H:OPPF deposition step. The hence obtained $\mu$ p-PPFs were then characterized by scanning electron microscope (SEM), X-ray photoelectron spectroscopy (XPS), time-of-flight secondary ion mass spectrometry (ToFSIMS)and water contact angle measurements (WCA). The lateral definition and selectivity of the $\mu$ p-PPFswas assessed via adsorptionof a fluorescent protein, which binds selectively to the amino-rich parts of the surfaces, as followed by microarray scanner analysis.

\section{EXPERIMENTAL SECTION}

\subsection{Preparation of the $\mu \mathrm{p}-\mathrm{PPF}$}

Deposition was performed in a cylindrical symmetric plasma reactor where the two plane parallel electrodes $(30 \mathrm{~cm}$ in diameter) are separated by a g lass ring $(5 \mathrm{~cm}$ in height). The upper electrode contains a gas showerhead, while the chamber is pumped through a central opening below the lower electrode, which is coupled to the RF generator enabling well-defined deposition conditions [14]. A capacitive-coupled field with $13.56 \mathrm{M} \mathrm{Hz}$ radiofrequency excitation was used to power a plasma of $\mathrm{C}_{2} \mathrm{H}_{4} / \mathrm{NH}_{3}$ and $\mathrm{C}_{2} \mathrm{H}_{4} / \mathrm{CO}_{2}$ mixtures to deposit the amino-containing (a-C:H:N) and oxygen-containing (a-C:H:O) films, respectively. After the deposition of nominally $5 \mathrm{~nm}$ of a-C:H:N,samples wereexposed to ambient air and a precision plain weave textile mesh (SEFAR PET 1500 36/92-90W PW) with $183 \mu \mathrm{m} \times 183 \mu \mathrm{m}$ square openings, and nominal thread diameter of $90 \mu \mathrm{m}$, was placed 
onto samples. This mesh was used as mask in direct contact with the a-C:H:N-coated sample during the deposition of a-C:H:O of nominally 5-10nm thickness (Figure 1).

The highly stable and previously establisheda-C:H:N coating [35], comprising 2 at $\%$ of $\mathrm{NH}_{2}$ groups, was prepared according to the following conditions: $\mathrm{NH}_{3}$ and $\mathrm{C}_{2} \mathrm{H}_{4}$ flow rates of $7 \mathrm{sccm}$ (gas ratio 1:1), pressure of $10 \mathrm{~Pa}$, and power of $50 \mathrm{~W}$ for $40 \mathrm{~s}$. Onto this stable base layer, an oxygen-containing a-C:H:O coatingwas deposited according toconditions that were optimized in previous studies [36,37]: $\mathrm{CO}_{2}$ and $\mathrm{C}_{2} \mathrm{H}_{4}$ flow rates of 24 and $4 \mathrm{sccm}$ respectively (gas ratio 6:1), pressure of $10 \mathrm{~Pa}$, and power of $30 \mathrm{~W}$ for 5 min.Glass slides, Si wafers and Ag-coated glass slides were used as substrates in this study, and were previously cleaned by Ar plasma (10 min, $50 \mathrm{~W}, 10 \mathrm{~Pa})$.
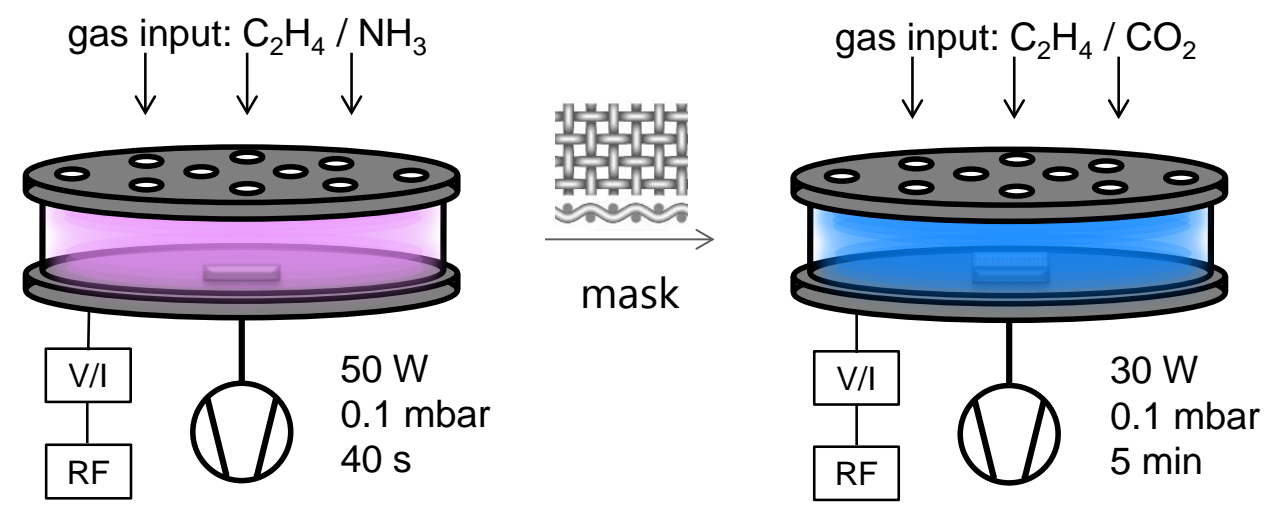

Figure 1.Schematic illustration of the preparation of the $\mu$ p-PPFs. The deposition of the second a-C:H:O layer is carried out through a polymeric mesh.

\subsection{Surface characterization}

\subsubsection{SEM analyses}

Scanning electron microscopy (Hitachi S-4800)was performed in order to visualize the patterning at the surface of Ag-coated glass slides. Note that a $\sim 50$ nmsilver layer was used to dissipate the surface charge, and may generate secondary electrons to enhance the pattern imaging [15].Analyses were performed using an acceleration voltage of $2 \mathrm{kV}$ at a current of $11 \mu \mathrm{A}$. Pictures were taken with 100x magnification.

\subsubsection{XPS analyses}

The chemical composition of the surfaces was analyzed by X-ray photoelectron spectroscopy (XPS) measurements using a Scanning XPS Microprobe (PHI VersaProbe II spectrometer, Physical Electronics) with monochromatic Al Ka radiation $(1486.6 \mathrm{eV})$. The operating pressure of the XPS analysis chamber was below $5 \times 10^{-7} \mathrm{~Pa}$ during the measurements. The spectra were collected at photoemission take 
off angles of $45^{\circ}$ (with respect to the sample surface) and using a focused X-ray beam with a diameter of $20 \mu \mathrm{m}$. Survey scan spectra $(0-1100 \mathrm{eV})$ were acquired with an energy step width of $0.8 \mathrm{eV}$, acquisition time of $160 \mathrm{~ms}$ per data point and analyzer pass energies of $187 \mathrm{eV}$. Region scan spectra were acquired with an energy step width of $0.125 \mathrm{eV}$, an acquisition time of $1.2 \mathrm{~s}$ per data point and analyzer pass energies of $29 \mathrm{eV}$. X-ray excited secondary electron images were done on $1 \mathrm{~mm}$ x $1 \mathrm{~mm}$ area, thanks to a focused $X$-ray beam with a size of $9 \mu \mathrm{m}$.In addition, also chemical mapping for carbon and oxygen (with pass energy of $117 \mathrm{eV}$ ) was performed using an X-ray beam with a diameter of $20 \mu \mathrm{m}$. Obtained spectra were rescaled by shifting the spectra relative to the aliphatic carbon at $285.0 \mathrm{eV}$ to account for charging effects. Curve fitting (least-squares fit routines) with CasaXPS software version 2.3.16 was employed to calculate the atomic concentrations.

\subsubsection{ToF-SIMSimaging}

Time of flight secondary ions mass spectrometry (ToF-SIMS.5, Iontof) was performed to analyze the laterally-resolved elemental and molecular composition of the topmost monolayers of the $\mu$ p-PPFssamples. A $50 \mathrm{keV} \mathrm{Bi}_{3}{ }^{++}$primary ions focused beam was used in high mass resolution mode $(\mathrm{M} / \Delta \mathrm{M} \sim 7000)$. A gentle sputtering with a low energy $\mathrm{Cs}^{+}$beam $\left(250 \mathrm{eV}, 1000 \times 1000 \mu^{2}\right)$ was simultaneously used to boostthe ionization yield in the negative polarity. Secondary ions image data, containing the full compositional spectrum in the mass range 1-500 mu for each pixel, were acquired with ultra-high sensitivity ( $\sim \mathrm{ppm})$ on randomly selected $500 \times 500 \mu \mathrm{m}^{2}$ areas with a $256 \times 256$ pixels $^{2}$ resolution.

\subsubsection{Water Contact Angle measurements}

Water contact angle (WCA) was measured on freshly prepared- and aged-samples. For the aging study, samples were stored in air or in distilled water at $20^{\circ} \mathrm{C}$. To determine the WCA, at least 3 drops $(2 \mu \mathrm{L})$ of water (CHROMASOLV®,for HPLC, Sigma Aldrich) were deposited onto the surface and analyzed using the software controlled drop shape analyzer(DSA25, Krüss).

\subsection{Spatially-defined protein adsorption \\ 2.3.1. Immobilization of green fluorescent protein}

The Y-tagged version of GFP (Y1-GFPuv), engineered to be a substrate for tyrosinase, was produced recombinant in E. coli and purified by affinity chromatography as reported. [38]Tyrosinase from Agaricusbisporus(T3824, $\geq 1000$ unit per mg) was purchased fromSigma 
Aldrich (Buchs, Switzerland). Reaction mixtures that contain $1 \mathrm{mg} / \mathrm{mLof}$ the genetically modified green fluorescent protein Y1-GFPuv were prepared in $100 \mathrm{mM}$ potassium phosphate buffer at pH 6.8 and the reaction was started by adding $0.1 \mathrm{mg} / \mathrm{mLty}$ rosinase immediately before the coating process. $200 \mu \mathrm{L}$ of the reaction mixture was dispensed on the $\mu \mathrm{p}$-PPF in form of a freestanding droplet, in a humid atmosphere to avoid evaporation of the drop,and the exposure was carried out at room temperature for $3 \mathrm{~h}$. Contact time in between plasma coating and protein-containing solution was reduced to minimize aging effects. After exposure, samples were thoroughly rinsed three times in ultrapure water for $10 \mathrm{~min}$ to remove loosely bound protein molecules. Samples were thenair-dried before imaging. The concentration of Y1GFPuv was determined either by the wavelength shift during dye-protein complexation with the Bradford method [38,39] using bovine serum albumin as standard, or simply using the extinction coefficient $27000 \mathrm{M}^{-1} \mathrm{~cm}^{-1}$ and a calculated molecular mass of $27862 \mathrm{~g} \mathrm{~mol}^{-1}$.

\subsubsection{Fluorescence imaging of the surface}

The presence of green fluorescence on the surfaces was visualized with a LS Reloaded Microarray Scanner (Tecan, Switzerland) equipped with a lasers at $488 \mathrm{~nm}$ and $532 \mathrm{~nm}$ filters). Imaging conditions included a gain value of 160 and a spatial resolution of $4 \mu \mathrm{m}$.

\section{RESULTS \& DISCUSSION}

\subsection{Surface characterization}

In order to assess the spatial distribution of the different chemical functionalitiesonthe $\mu$ p-PPFs, different surface analytic tools were used. To visualize the micropattern, SEM analyses were carried out on a selection of different PPFs: a homogeneous layer of a-C:H:N, a homogeneous layer of a-C:H:O, the pattern-free reference multilayer structure where the a$\mathrm{C}: \mathrm{H}:$ Olayer was deposited onto the a-C:H:N layer, andthe $\mu \mathrm{p}-\mathrm{PPF}$ where the second a-C:H:O layer was deposited through the mask onto the a-C:H:N layer. While the a-C:H:N, a-C:H:O and double-layer PPFs appear to be homogeneous (Figure 2a, 2b and 2c)aregular micropatterning is revealed for the $\mu \mathrm{p}-\mathrm{PPF}$ (Figure $2 \mathrm{~d}$ ), due to a morphological contrast. Moreover, from thisSEM analysis, it is possible to give some geometrical information about the prepared pattern: the a-C:H:O squares are of dimension $206 \pm 3 \mu \mathrm{m}$ and are separated by a58 $\pm 2 \mu$ mwide grid of the maskedarea that corresponds to the a-C:H:N nano-PPF. In comparison with the nominal mesh opening, the coated area is larger; this is due to the lateral diffusion of the film-forming species created in the plasma chamber while depositing a-C:H:O film before their adsorption at the surface of the a-C:H:N layer. 


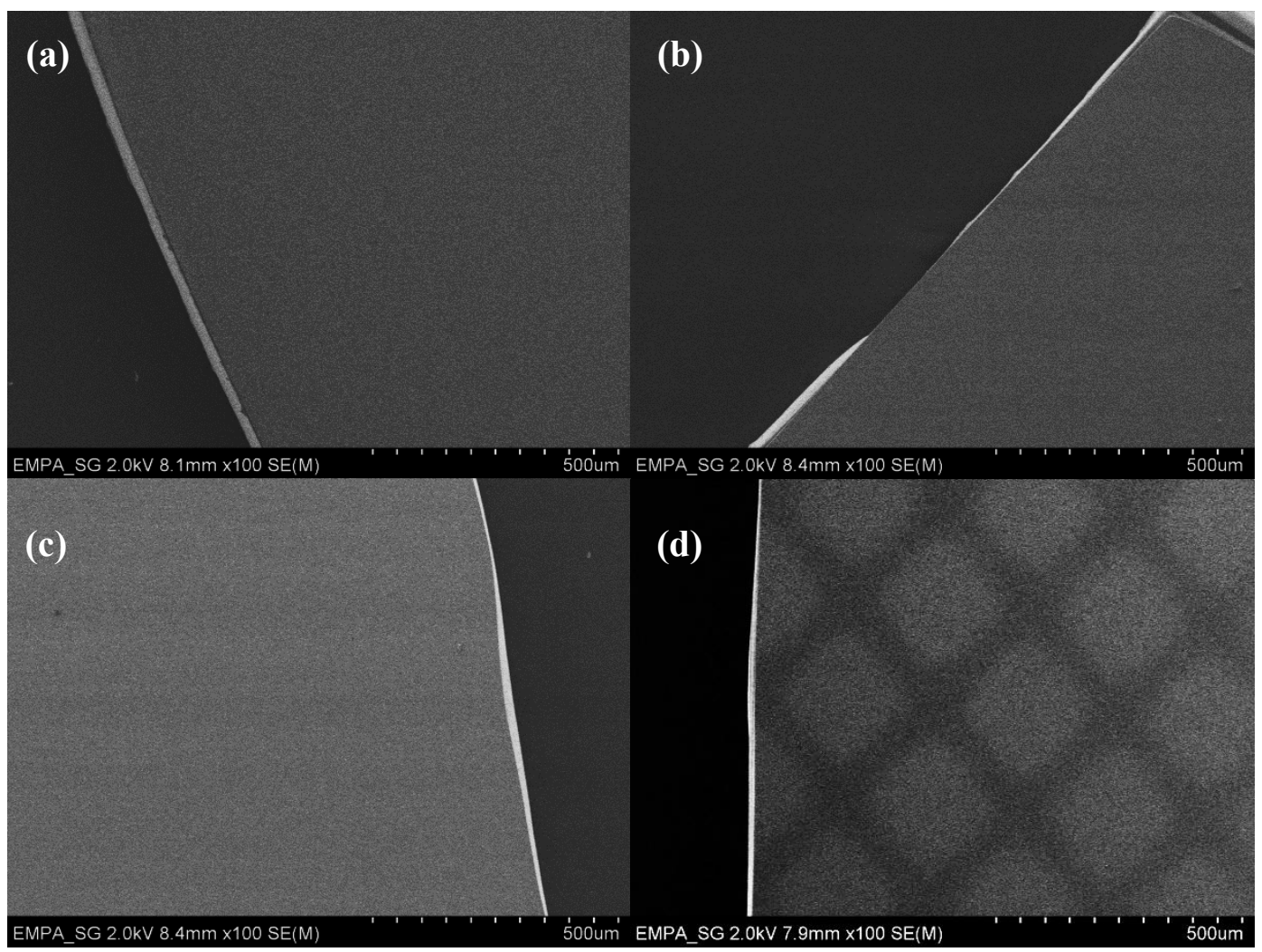

Figure 2. SEM imaging of samples with(a) a-C:H:N, (b) a-C:H:O, (c) a-C:H:O deposited onto a-C:H:N without any mask and (d) a-C:H:O deposited onto a-C:H:N using a mask to produce the $\mu$ p-PPFs.

Thanks to XPS mapping, it was possible to image and quantify the chemical composition of the surface. Scanning X-ray imaging (SXI, X-ray excited secondary electron image) showed a higher signal on the grid lines than in the squares between them (Figure 3a). The $[\mathrm{O}] /[\mathrm{C}]$ and $[\mathrm{N}] /[\mathrm{C}]$ ratios obtained in the squares are comparable to the ratios obtained for the reference homogeneous a-C:H:O coating (Figure $3 \mathrm{~b}$ and Table 1), showing that the a$\mathrm{C}: \mathrm{H}: \mathrm{O}$ coating was successfully deposited through the mask and has a similarsurface composition as a monolayer of the same coating. On the contrary, the chemical ratio observed on the masked area doesnot correspond to any of the monolayer-references. The $[\mathrm{O}] /[\mathrm{C}]$ ratio is higher $(31.7 \%)$ than expected $(10.3 \%$ for a-C:H:N) and the $[\mathrm{N}] /[\mathrm{C}]$ ratio is lower $(6.1 \%$ compared to the $18.5 \%$ for a-C:H:N). Thus, the first layer, i.e. the a-C:H:N monolayer, may have been oxidized while depositing the a-C:H:O at the grid position of the textile contact mask. Note that the used mask is made of a woven monofilament mesh allowing for some access of plasma species below the grid lines.Imaging XPS allowsvisualizing the chemical differentiation of the patterning; in particular, the atomic composition in carbon and oxygen is relevant (supporting information Figures S1 and S2) showing that the less-functionalized 
carbon amount increases in the square area and decreases in the grid area, and in analogy, the oxygen amount decreases in the square area and increases in the grid area.

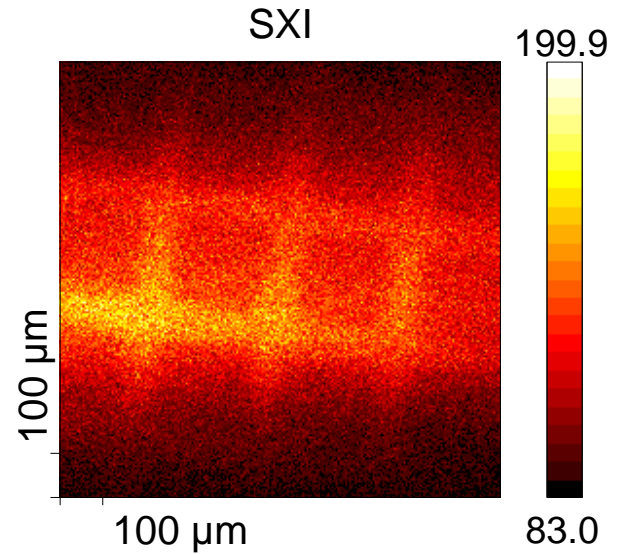

(a)

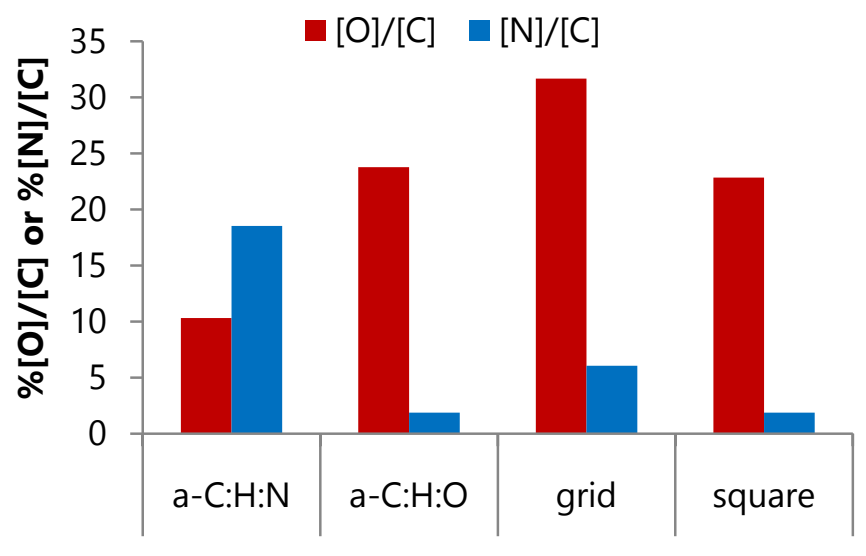

(b)

Figure 3. XPS analyses of $\mu$ p-PPF: (a) X-ray excited secondary electron image (SXI) and (b) $[\mathrm{O}] /[\mathrm{C}]$ and $[\mathrm{N}] /[\mathrm{C}]$ ratios in comparison with the homogeneous reference a-C:H:N and a$\mathrm{C}: \mathrm{H}: \mathrm{O}$ plasma polymer layers.

Table 1. Quantification by XPS of the elemental composition of a-C:H:N and a-C:H:O homogeneous layers listed as grid- and square- areas of the $\mu \mathrm{p}-\mathrm{PPF}$.

\begin{tabular}{lccccccc}
\hline & $\% \mathbf{C 1 s}$ & $\% O 1 s$ & $\% N 1 s$ & $\% S i 2 p$ & {$[\mathrm{O}] /[\mathrm{C}]$} & {$[\mathrm{N}] /[\mathrm{C}]$} & $([\mathrm{O}]+[\mathrm{N}]) /[\mathrm{C}]$ \\
\hline a-C:H:N & 76.7 & 7.9 & 14.2 & 1.1 & $10.3 \%$ & $18.5 \%$ & $28.8 \%$ \\
a-C:H:O & 79.6 & 18.9 & 1.5 & - & $23.8 \%$ & $1.9 \%$ & $25.7 \%$ \\
grid area & 72.6 & 23.0 & 4.4 & - & $31.7 \%$ & $6.1 \%$ & $37.7 \%$ \\
square area & 80.1 & 18.3 & 1.5 & - & $22.8 \%$ & $1.9 \%$ & $24.7 \%$ \\
\hline
\end{tabular}

A ToF-SIMS study was carried out to complementthe results obtained with XPS, and obtain further molecular information athigh lateral resolution. Firstly, secondary ion mass spectra were obtained on the two homogeneous reference layers; a-C:H:N and a-C:H:O, in order to identifythe respective characteristic fragments of the two types of plasma polymers. The referencedouble layer consisting of a homogeneous a-C:H:Olayer on $\mathrm{t}$ op of a homogeneous a-C:H:N layer was also analyzed. The fragments observed in the bilayer configuration were exactly the same as fora single homogeneous a-C:H:O coating, confirming that the bottom layer has no influence on surface chemistry and that the top layerof the double layer is chemically identicalto a single a-C:H:O layer. Then, $\mu$ p-PPF was analyzed and secondary ionsimages corresponding to the respective characteristic fragments as determined 
on the homogeneous samples were extracted.Figure 4 shows the spatial distribution of selected fragments at the surface of $\mu$ p-PPF (see also supplementary Figure S3). Note that to improve the signal over noise ratio, the contribution of some fragments of similar chemical originwere summed into groups as illustrated in Table 2.

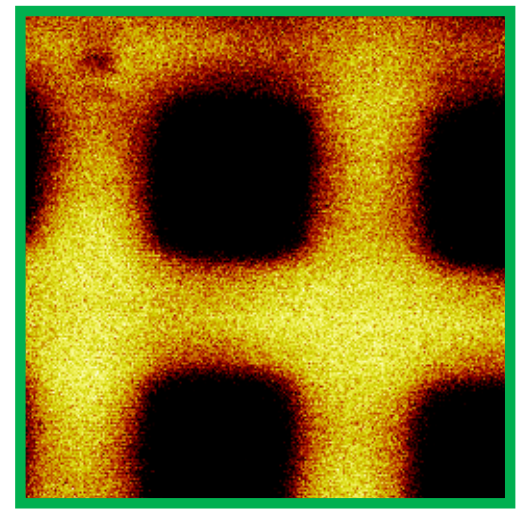

$\mathrm{CxNy}$

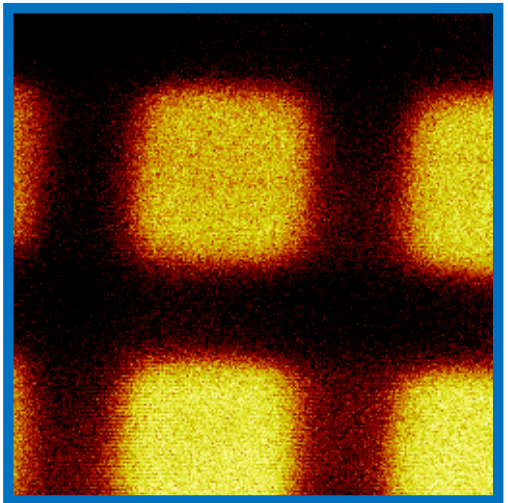

$\mathrm{Cx}$

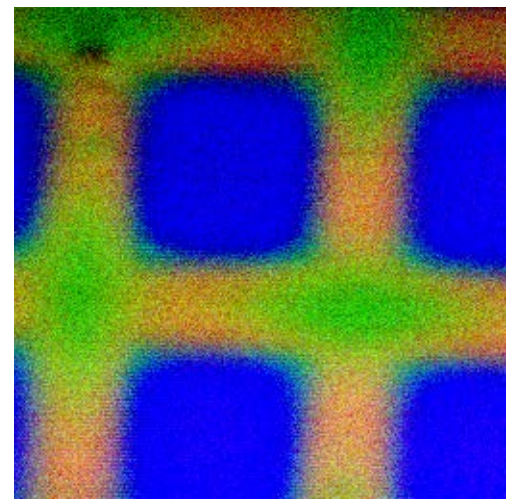

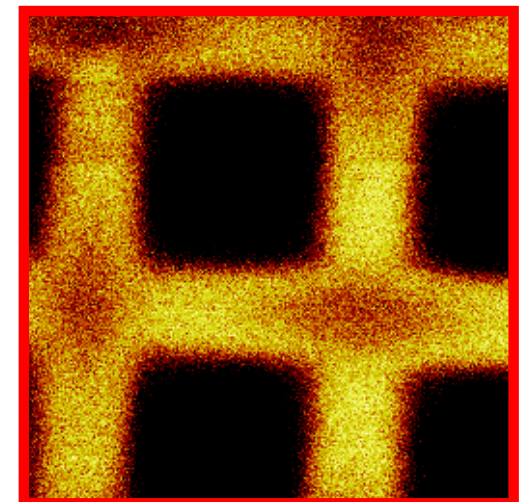

$\mathrm{CHxNO}$

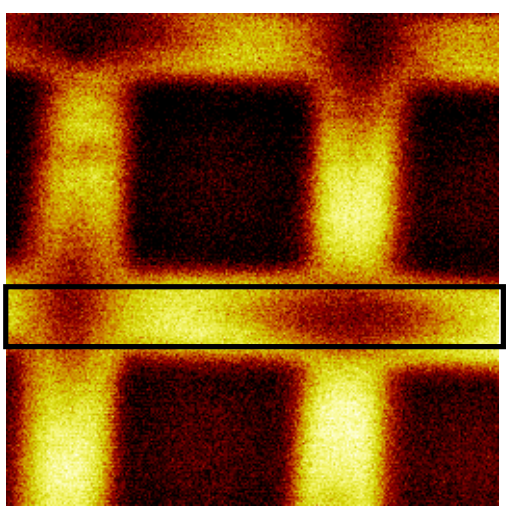

$\mathrm{CxHyOz}$

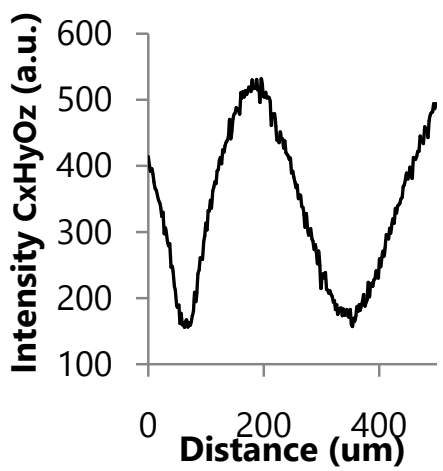

\section{RGB-COMPOSITE IMAGE}

\section{CxHyOz PROFILE}

Figure 4: ToF-SIMS negative secondary ions images (Field of view $500 \mathrm{x} 500 \mu \mathrm{m}^{2}$ ) of the micro-patterned PPF deposited onto Si-wafer (yellow areas correspond to higher signals, brown areas correspond to lower signals). The RGB-composite image was generated with $\mathrm{CHxNO}$ in red, $\mathrm{CxNy}$ in green and $\mathrm{Cx}$ in blue. The $\mathrm{CxHyOz}$ Profile was carried out by extracting RGB information from an area of image $\mathrm{CxHyOz}$ using the software ImageJ. 
Table 2. Fragments measured by ToF-SIMS during the analysis of the $\mu$ p-PPF films. For ToFSIMS image analysis, only group names will be given, but all of these fragments were considered.

\begin{tabular}{llllll}
\hline Groups & Fragments & $\mathbf{m} / \mathbf{z}$ & Groups & Fragments & $\mathbf{m} / \mathbf{z}$ \\
\hline $\mathbf{C x N y}$ & ${ }^{13} \mathrm{CN}^{-}$ & 27.0056 & $\mathbf{C x}$ & $\mathrm{C}^{-}$ & 12.0019 \\
& $\mathrm{C}_{3} \mathrm{~N}^{-}$ & 50.0124 & $\mathrm{C}_{2}^{-}$ & 24.0026 \\
& $\mathrm{C}_{3} \mathrm{~N}_{2}^{-}$ & 64.0189 & $\mathrm{C}_{4}^{-}$ & 48.0061 \\
& $\mathrm{C}_{2} \mathrm{~N}_{3}^{-}$ & 66.0092 & $\mathrm{C}_{6}^{-}$ & 72.0047 \\
& $\mathrm{C}_{5} \mathrm{~N}^{-}$ & 74.0087 & $\mathrm{C}_{7}^{-}$ & 84.0038 \\
$\mathbf{C x H y N z}$ & $\mathrm{C}_{2} \mathrm{H}_{2} \mathrm{~N}^{-}$ & 40.0214 & $\mathrm{C}_{8}^{-}$ & 96.0044 \\
& $\mathrm{C}_{3} \mathrm{H}_{2} \mathrm{~N}^{-}$ & 52.0222 & $\mathrm{C}_{10}{ }^{-}$ & 120.0125 \\
& $\mathrm{C}_{4} \mathrm{HN}^{-}$ & 63.0157 & $\mathbf{C x H y}$ & $\mathrm{C}_{3} \mathrm{H}^{-}$ & 37.0096 \\
$\mathbf{C x H y O z}$ & $\mathrm{C}_{2} \mathrm{HO}^{-}$ & 41.0076 & $\mathrm{C}_{5} \mathrm{H}^{-}$ & 61.0094 \\
& $\mathrm{C}_{2} \mathrm{H}_{3} \mathrm{O}^{-}$ & 43.0198 & & $\mathrm{C}_{6} \mathrm{H}^{-}$ & 73.0162 \\
& $\mathrm{CHO}_{2}^{-}$ & 44.9929 & & $\mathrm{C}_{7} \mathrm{H}^{-}$ & 85.0089 \\
& $\mathrm{C}_{3} \mathrm{H}_{3} \mathrm{O}^{-}$ & 55.0234 & & $\mathrm{C}_{8} \mathrm{H}^{-}$ & 97.0175 \\
& $\mathrm{C}_{3} \mathrm{H}_{5} \mathrm{O}^{-}$ & 57.0373 & & $\mathrm{C}_{10} \mathrm{H}^{-}$ & 121.0212 \\
& $\mathrm{C}_{2} \mathrm{H}_{3} \mathrm{O}_{2}^{-}$ & 59.0040 & & $\mathrm{C}_{12} \mathrm{H}^{-}$ & 145.0251 \\
& $\mathrm{C}_{4} \mathrm{H}_{3} \mathrm{O}^{-}$ & 67.0237 & & $\mathrm{C}_{14} \mathrm{H}^{-}$ & 169.0261 \\
& $\mathrm{C}_{3} \mathrm{HO}_{2}^{-}$ & 68.9884 & $\mathbf{C H x N O}$ & $\mathrm{CNO}^{-}$ & 42.0012 \\
& $\mathrm{C}_{3} \mathrm{H}_{3} \mathrm{O}_{2}^{-}$ & 71.0076 & $\mathrm{CH}_{2} \mathrm{NO}^{-}$ & 44.0142 \\
\hline
\end{tabular}

A composite false color image (Figure 4) was generated where the Cx contribution is given in blue, the $\mathrm{CxNy}$ contribution in green and $\mathrm{CHxNO}$ contribution in red, visualizing the chemistries at the surfaces of the $\mu$ p-PPF.

$\mathrm{Cx}$ and $\mathrm{CxHyfragments} \mathrm{were} \mathrm{found} \mathrm{to} \mathrm{be} \mathrm{more} \mathrm{abundant} \mathrm{in} \mathrm{the} \mathrm{square} \mathrm{areas,} \mathrm{which} \mathrm{is}$ in agreement with the carbon coverage obtained by XPS. Thus, the a-C:H:O coating released more hydrocarbon secondary ions than the a-C:H:N coating. Other fragments, particularly,CxNy, $\mathrm{CxHyNz}, \mathrm{CxHyOz}$ and $\mathrm{CHxNOaremainly} \mathrm{observed} \mathrm{in} \mathrm{the} \mathrm{maskedarea,} \mathrm{as}$ expected. Surprisingly though, Figure 4 (and Figure S3) also reveals that signals are inhomogeneous acrossthe masked area, namelyCxNyspecies seem to be more concentrated in the crossingarea of the fiber mesh, whereas $\mathrm{CxHyNz}, \mathrm{CxHyOz}$ and $\mathrm{CHxNO}$ are mainly presentin the connecting linear part of the pattern, leading to a lateral chemical gradient inside 
the maskedarea. This result can be explained by the fact that the mask was a polymeric fiber mesh with a weaving structure providing a better mechanical contact to the substrate at locations where the filamentscross, and therefore less lateral diffusion duringdeposition ofaC:H:O.In the free lying part of the mesh fibers, a limited amount ofplasma species can still interact with the a-C:H:N base layer.The chemical gradient is visible in the imaging scans. We can plot an oxygen profile along the gradient provided the $\mathrm{CxHyOz}$ fragments, showing that the variation in chemical composition is congruent with the textile binding pattern used in the fiber mesh mask.

For plasma polymer coatings, stability in air and in water is an important factor to be considered. First investigation on evolution of wetting properties over time was carried out by measuring the static water contact angle (WCA) in regular intervals after deposition of the $\mu$ pPPF. As references, the homogeneous layers of a-C:H:N and a-C:H:O yielded an initial WCA of $\sim 55^{\circ}$ and $\sim 46^{\circ}$ respectively. Samples have been stored in air or in water for one week. Samples stored in air were directly measured after their storage process, whereas samples stored in water were first blow dried with compressed air at their surface and then measured immediately. Figure 5 shows the static water contact angles measured for the $\mu$ p-PPFsstored in air or in water. Note that the drop size was much bigger $(2-3 \mathrm{~mm}$ diameter) than the pattern size (264 $\pm 5 \mu \mathrm{m}$ width), and thus, both square and grid areas are simultaneouslymeasured as an effective averaged value. This effective WCA shows a similar variation statistics as the WCA measured on plain reference coatings and can thus be used as a measure for the effective aging of the $\mu \mathrm{p}-\mathrm{PPF}$.

When stored in air the WCA of the a-C:H:N reference remains unchanged for the duration of one week, and the WCA of the a-C:H:O reference is approximately the same within error of the measurement indicating the stability of these coatings. The $\mu$ p-PPF curve may be interpreted as a small trend toincrease the WCA over one week (starting from $45^{\circ}$ to reach $\sim 50^{\circ}$ ). This observation might be explained by the fact that some restructuring may occur at the surface of the film.

The effective water contact angle of the $\mu \mathrm{p}$-PPF during the first few days are globally close to the wetting properties of the a-C:H:O reference. This results could be explained by the fact that the a-C:H:O coating covers the majority of the surface of $\mu$ p-PPF (square-to-grid area of $\sim 1.6$ ), and was added at the end of the coating process, and thus, can be considered as a top layer. 
After storage in water(and subsequent briefblow drying), a decrease of the WCAwas observed for references a-C:H:N and a-C:H:O. This might be related to a slight hydration of the surface, and some water molecules can be still bound onto polar chemical groups, such as carboxylic acid, hydroxyl or amino groups, through hydrogen bonding. Considering the $\mu$ pPPF, a slightly higher WCA was measured compared to the reference a-C:H:O, but WCA decreased with the same trends as observed for the a-C:H:O reference, showing that the wetting properties of the bilayer coating essentiallyfollows the top layer.

Finally, thesmallaging effect of $\mu$ p-PPF and both reference coatings when immersed in water for 1 day suggest that it is safe to usethese coatings for the GFP adsorption test requiring less than four hours water exposure.
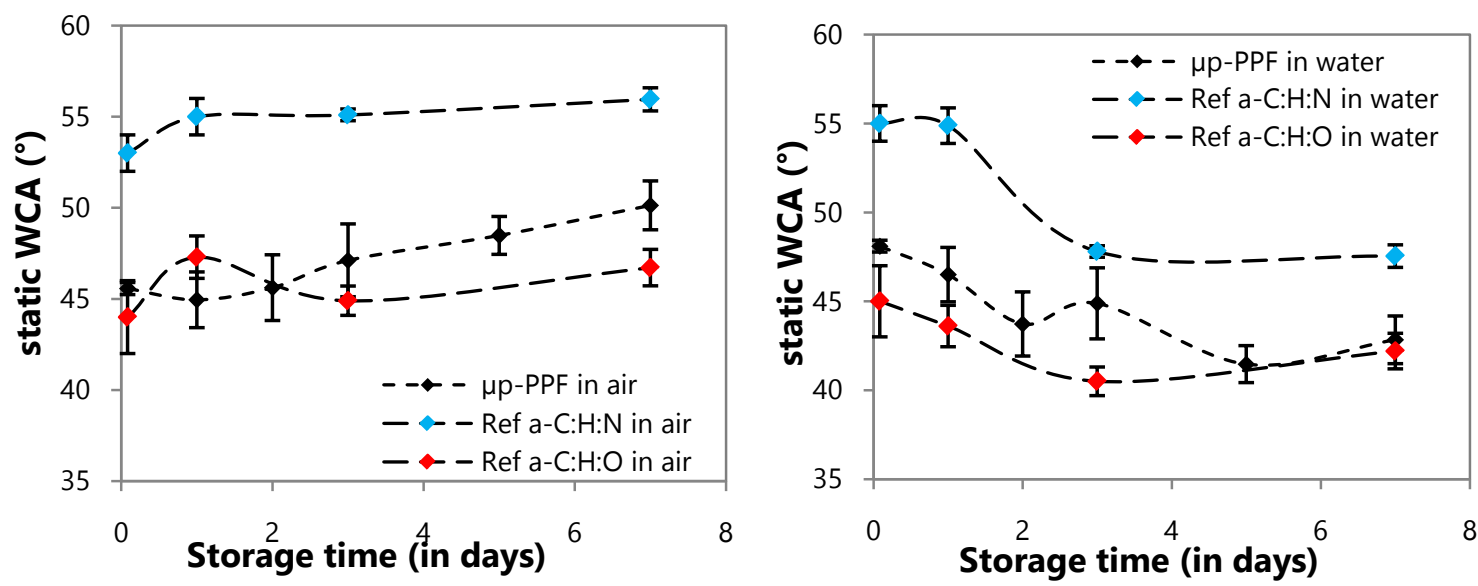

Figure 5.Static water contact angles of $\mu$-PPFsstored in air and in water.

\subsection{Spatially-defined protein deposition}

Finally, the functionality in view of future bio-sensing applicationsof the micropatterned surface has been tested by immersing the $\mu$ p-PPF in a solution that contains GFP, and, tyrosinaseto covalently bind GFP onto amino groups. Indeed, a similar approach couldbe used later to adsorb enzymes or antibodies at the surface of the $\mu p$-PPF, leading to new biosensors. GFP was chosen because of its fluorescence that can be readilymeasured after immersion and a cleaning step.

Fluorescence imaging of the $\mu \mathrm{p}-\mathrm{PPF}$ surface before and after immersion in GFP shows the preferential spatial distribution of the GFP (Figure 6). T he proteins are specifically adsorbingon the masked amino-containing areasof the $\mu$ p-PPF (see supplementary Figure S4).

A first possible explanation for this selective adsorption may be related to the surface charge. Indeed, GFP has an isoelectric point of $\sim 5$ and thus carries a net negative charge when incubated in phosphate buffer at $\mathrm{pH}$ 6.8. GFP will thus preferentially adsorb to positively 
charged surfaces such as the amino-functionalized regions of the $\mu \mathrm{p}-\mathrm{PPF}$. Moreover, in this study, as we used a-C:H:N and a-C:H:O coating, it ispossible to sensethe different chemical affinities, i.e. $-\mathrm{NH}_{2}$ vs. $-\mathrm{CO}(\mathrm{X})$, where $\mathrm{X}$ can be $-\mathrm{OH}$, -O-Alkyl or $-\mathrm{Alkyl}$ for example, by using suchenzyme. Namely, tyrosinaseenhances the oxidation of the tyrosine of the Y-tag of the engineered proteins, enhancing a chemical coupling with the amino groups present at the surface of the substrate $[38,40]$. Thus, a combination of adsorption and enzymatic immobilizationoccurs in the maskeda-C:H:Narea $[38,39]$.

Furthermore, it a ppears that the fluorescence intensity is much larger at the grid crossing areas, demonstrating an even stronger adsorption of the proteins at these specific locations of the well masked a-C:H:N surface. Moreover, due to the use of tyrosinase, a certain amount of GFP would have been covalently bound onto the amine groups [40]. As revealed by ToF-SIMS, these regions correspond to a pure non-oxidized a-C:H:N film, whereas the rest of the grid is composed of $\mathrm{CxHyOz}$ and $\mathrm{CHxNO}$. This demonstrates that GFP not only adsorbs on pure a-C:H:N but can also adsorb on the oxidized regions, although to a lower degree. By creating smooth oxidation gradients within $\mu \mathrm{p}-\mathrm{PPF}$ the interaction of proteins with surfaces can be specially controlled and might be of interest for bio-sensing applications.

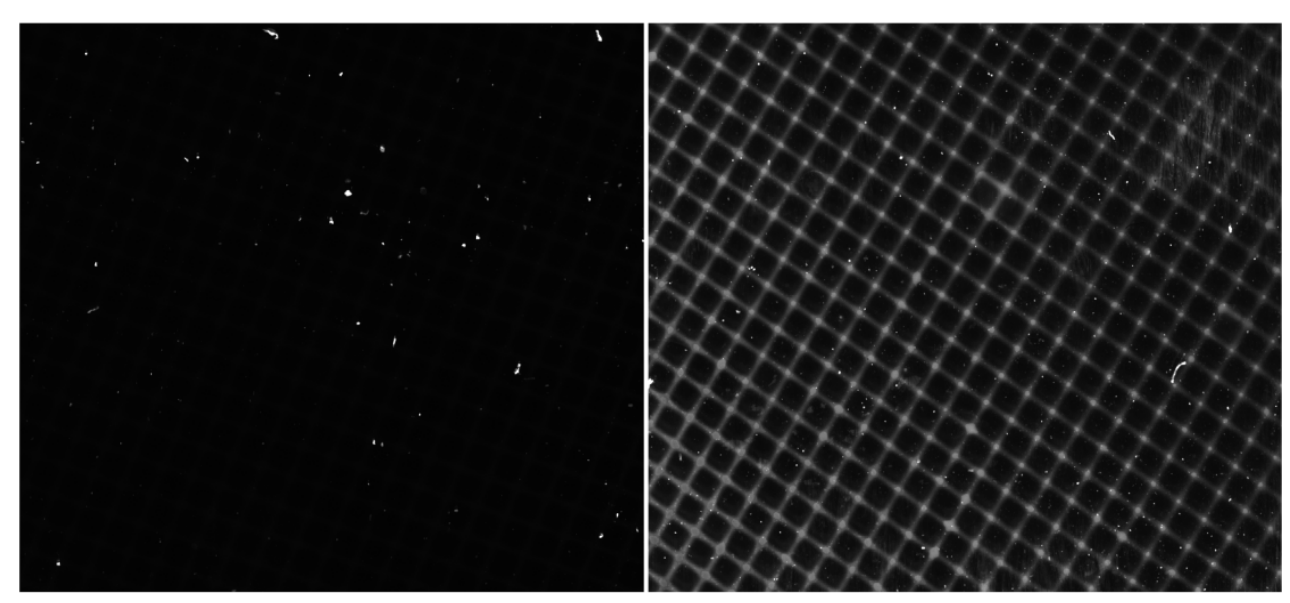

(a)

(b)

Figure 6. Microarray scanner imaging of the $\mu$ p-PPF (a) before and (b) after GFP adsorption. 


\section{CONCLUSION}

In this study, the feasibility of an all-plasma process to design a micro-patterned plasma polymer film( $\mu \mathrm{p}-\mathrm{PPF})$ with both oxygen-rich and amine-rich regions was demonstrated. SEM, XPS and ToF-SIMS surface characterization of the $\mu$-PPF revealed the lateral distribution of the surface chemical groups: amine groups were located in the grid area, whereas the oxygen-containing groups provided by the second plasma deposition where in the square area.Moreover, the existence of a lateral chemical gradient within the masked aminebased area was found congruent with the fiber mesh maskmechanical contact. As a simple technique, a textile web mesh was used as a mask allowing variablecontact geometries withthe surface and therefore locally controlled lateral diffusion of plasma species during plasma treatment. Over micrometer distances, local lateral chemical gradients were observed.

In summary, the here presented $\mu$ p-PPFscombine vertical chemical gradientsfrom amino-containing to oxygen-containing PPF, and lateral chemical gradients from aminocontaining PPF to oxidized PPF. This provides an interesting template surface for the development of future multi-functional affinity bio-sensors.

The stability of the $\mu$ p-PPF can be inferred from a nearly constant water contact angle over several days.In addition, the selectivity for protein adsorption onto the $\mu$ p-PPF was successfully tested by exposure to a solution that contains a fluorescent proteinGFP, which was observed to adsorbsolelyon the nitrogen-containing areas.

\section{ACKNOWLEDGEMENTS}

The authors gratefully acknowledge Swiss National Science Foundation (SNSF, Bern) that funded this study under grant no. IZ73Z0_152661 (SCOPES). 


\section{REFERENCES}

[1] A. Javid, M. Kumar, L. Wen, S. Yoon, S.B. Jin, J.H. Lee, J.G. Han, Surface energy and wettability control in bio-inspired PEG like thin films, Mater. Design 92 (2016) 405413.

[2] M. Kumar, J.X. Piao, S.B. Jin, J.H. Lee, S. Tajima, M. Hori, J.G. Han, Low temperature plasma processing for cell growth inspired carbon thin films fabrication, Arch. Biochem. Biophys. 605 (2016) 41-48.

[3] J. X. Piao, M. Kumar, A. Javid, S. Yoon, J.H. Lee, J.G. Han, Pulsed DC-plasma sputtering induced synthesis of hydrogenated carbon thin films for L-929 cell cultivation, Surf. Coat. Technol. (2016) in press

[4] S. David, C. Polonschii, C. Luculescu, M. Gheorghiu, S. Gaspar, E. Gheorghiu, Magneto-plasmonic biosensor with enhanced analytical response and stability, Biosens. Bioelectron.63 (2014) 525-532.

[5] M. Heuberger, T.E. Balmer, The transmission interferometric adsorption sensor, J. Phys. D: Appl. Phys. 40 (2007) 7245-7254.

[6] E. Wijaya, C. Lenaerts, S. Maricot, J. Hastanin, S. Habraken, J.-P. Vilcot, R. Boukherroub, S. Szunerits, Surface plasmon resonance-based biosensors:From the development of different SPR structures to novel surface functionalization strategies, Curr. Opin. Solid St. M. 15 (2011) 208-224.

[7] E. Reimhult, F. Höök, Design of surface modifications for nanoscale sensor applications, Sensors 15 (2015) 1635-1675.

[8] D. Falconnet, A. Koenig, F. Assi, M. Textor, A combined photolithographic and molecular-assembly approach to produce functional micropatterns for applications in the biosciences, Adv. Funct. Mater. 14 (2004) 749-756.

[9] D. Falconnet, D. Pasqui, S. Park, R. Eckert, H. Schift, J. Gobrecht, R. Barbucci, M. Textor, A novel approach to produce protein nanopatterns by combining nanoimprint lithography and molecular self-assembly, Nano Lett. 4 (2004) 1909-1914.

[10] V. Crivianu-Gaita, M. Thompson, Immobilization of Fab' fragments onto substrate surfaces: A survey of methods and applications, Biosens. Bioelectron. 70 (2015) 167-180.

[11] S. Guimond, U. Schütz, B. Hanselmann, E. Körner, D. Hegemann, Influence of gas phase and surface reactions on plasma polymerization, Surf. Coat. Technol. 205 (2011) S447-S450.

[12] D. Hegemann, E. Körner, K. Albrecht, U. Schütz, S. Guimond, Growth mechanism of oxygen-containing functional plasma polymers, Plasma Proc. Polym. 7 (2010) 889-898.

[13] D. Hegemann, E. Körner, N. Blanchard, M. Drabik, S. Guimond, Densification of functional plasma polymers by momentum transfer during film growth, Appl. Phys. Lett. 101 (2012) 211603.

[14] D. Hegemann, M. Michlicek, N.E. Blanchard, U. Schütz, D. Lohmann, M. Vandenbossche, L. Zajickova, M. Drabik, Deposition of Functional Plasma Polymers Influenced by Reactor Geometry in Capacitively Coupled Discharges, Plasma Process. Polym. 13 (2016) 279-286.

[15] L. Dai, H.J. Griesser, A.W.H. Mau, Surface modification by plasma etching and plasma patterning, J. Phys. Chem. B 101 (1997) 9548-9554. 
[16] V. Sciarratta, C. Oehr, C. Diegelmann, P. Löbmann, Influence of plasma functionalization of poly(propylene) with acrylic acid on the nucleation of $\mathrm{CaCO} 3$, Plasma Process. Polym. 1 (2004) 51-56.

[17] Q. Li, X. Han, J. Hou, J. Yin, S. Jiang, C. Lu, Patterning Poly(dimethylsiloxane) Microspheres via Combination of Oxygen Plasma Exposure and Solvent Treatment, J. Phys. Chem. B 119 (2015) 13450-13461.

[18] P.-L. Girard-Lauriault, F. Mwale, M. Iordanova, C. Demers, P. Desjardins, M.R. Wertheimer, Atmospheric pressure deposition of micropattern nitrogen-rich plasma-polymer films for tissue engineering, Plasma Process. Polym. 2 (2005) 263-270.

[19] M. Thomas, J. Borris, A. Dohse, M. Eichler, A. Hinze, K. Lachmann, K. Nagel, C.-P. Klages, Plasma printing and related techniques - Patterning of surfaces using microplasmas at atmospheric pressure, Plasma Process. Polym. 9 (2012) 1086-1103.

[20] E. Sardella, R. Gristina, R. d'Agostino, P. Favia, Chapter 13 - Micro- and Nanostructuring in Plasma Processes for Biomaterials : Micro- and Nano-features as Powerful Tools to Address Selective Biological Responses, in: R. D’Agostino, P. Favia, Y. Kawai, H. Ikegami, N. Sato, F. Arefi-Khonsari (Eds.), Advanced Plasma Technology, Weinheim, 2008, pp. 243-268.

[21] T.C. Shyu, P.F. Damasceno, P.M. Dodd, A. Lamoureux, L. Xu, M. Shlian, M. Shtein, S.C. Glotzer, N.A. Kotov, A kirigami approach to engineering elasticity in nanocomposites through patterned defects, Nat. Mater. 14 (2015) 785-789.

[22] A. George, T.M. Stawski, S. Unnikrishnan, S.A. Veldhuis, J.E. Ten Elshof, Micro and nanopatterning of func-tional materials on flexible plastic substrates via site-selective surface modification using oxygen plasma, J. Ma-ter. Chem. 22 (2012) 328-332.

[23] E. Sardella, P. Favia, R. Gristina, M. Nardulli, R. d'Agostino, Plasma-aided micro- and nanopatterning processes for biomedical applications, Plasma Process. Polym. 3 (2006) 456-469.

[24] A. Malainou, K. Tsougeni, K. Ellinas, P.S. Petrou, V. Constantoudis, E. Sarantopoulou, K. Awsiuk, A. Bernasik, A. Budkowski, A. Markou, I. Panagiotopoulos, S.E. Kakabakos, E. Gogolides, A. Tserepi, Plasma-assisted nanoscale protein patterning on Si substrate via colloidal lithography, J. Phys. Chem. A 117 (2013) 13743-13751.

[25] O. Kylian, A. Choukourov, H. Biedermann, Nanostructured plasma polymers, Thin Solid Films 548 (2013) 1-17.

[26] G. Singh, H.J.Griesser, K. Bremmell, P. Kingshott, Highly ordered nanometer-scale chemical and protein pat-terns by binary colloidal crystal lithography combined with plasma polymerization, Adv. Funct. Mater. 21 (2011) 540-546.

[27] D.-G. Choi, H.K. Yu, S.G. Jang, S.-M. Yang, Colloidal Lithographic Nanopatterning via Reactive Ion Etching, J. Am. Chem. Soc. 126 (2004) 7019-7025.

[28] A. Valsesia, P. Colpo, M.M. Silvan, T. Meziani, G. Ceccone, F. Rossi, Fabrication of nanostructured polymeric surfaces for biosensing devices, Nano Lett. 4 (2004) 10471050.

[29] E.J. Szili, S.A. Al-Bataineh, P. Ruschitzka, G. Desmet, C. Priest, H.J. Griesser, N.H. Voelcker, F.J. Harding, D.A. Steele, R.D. Short, Microplasmaarrays : A new approach for maskless and localized patterning of materials surfaces, RSC Adv.2 ( 2012) 1200712010.

[30] S.A. Al-Bataineh, R.D. Short, Protein patterning on microplasma-activated PEO-like coatings, Plasma Process. Polym. 11 (2014) 263-268. 
[31] D.J. Menzies, T. Gengenbach, J.S. Forsythe, N. Birbilis, G. Johnson, C. Charles, G. McFarland, R.J. Williams, C. Fong, P. Leech, K. McLean, B.W. Muir, One step multifunctional micropatterning of surfaces using asymmetric glow discharge plasma polymerization, Chem. Comm. 48 (2012) 1907-1909.

[32] G. Mishra, C.D. Easton, G.J.S. Fowler, S.L. McArthur, Spontaneously reactive plasma polymer micropatterns, Polymer 52 (2011) 1882-1890.

[33] B.W. Muir, A. Fairbrother, T.R. Gengenbach, F. Rovere, M.A. Abdo, K.M. McLean, P.G. Hartley, Scanning probe nanolithography and protein patterning of low-fouling plasma polymer multilayer films, Adv. Mater. 18 (2006) 3079-3082.

[34] K.S. Siow, L. Britcher, S. Kumar, H.J. Griesser, Plasma Methods for the Generation of Chemically Reactive Surfaces for Biomolecule Immobilization and Cell Colonization A Review, Plasma Process. Polym. 3 (2006) 392-418.

[35] M. Vandenbossche, M.I.Butron Garcia, U. Schütz, P. Rupper, M. Amberg, D. Hegemann, Initial Growth of Functional Plasma Polymer Nanofilms, Plasma Chem. Plasma Process. 36 (2016) 667-677.

[36] E. Körner, G. Fortunato, D. Hegemann, Influence of RF plasma reactor setup on carboxylated hydrocarbon coatings, Plasma Process. Polym. 6 (2009) 119-125.

[37] D. Hegemann, E. Lorusso, M.-I. Butron-Garcia, N.E. Blanchard, P. Rupper, P. Favia, M. Heuberger, M. Vandenbossche, Suppression of Hydrophobic Recovery by Plasma Polymer Films with Vertical Chemical Gradients, Langmuir 32 (2016) 651-654.

[38] G. Faccio, S. Senkalla, L. Thöny-Meyer, M. Richter, Enzymatic multi-functionalization of microparticles under aqueous neutral conditions, RSC Adv. 5 (2015) 22319-22325.

[39] M.M. Bradford, A Rapid and Sensitive Method for the Quantitation of Microgram Quantities of Protein Utilizing the Principle of Protein-Dye Binding, Anal.Biochem. 72 (1976) 248-254.

[40] G. Faccio, M.M. Kämpf, C. Piatti, L. Thöny-Meyer, M. Richter, Tyrosinase-catalyzed site-specific immobilization of engineered C-phycocyanin to surface, Sci. Rep. 4 (2014) 5370 . 